\title{
Anthropogenic influence on extreme precipitation over global land areas seen
}

\section{in multiple observational datasets}

\author{
Gavin D. Madakumbura ${ }^{1^{*}}$, Chad W. Thackeray ${ }^{1}$, Jesse Norris ${ }^{1}$, Naomi Goldenson ${ }^{1}$ \\ and Alex Hall ${ }^{1}$ \\ ${ }^{1}$ Department of Atmospheric and Oceanic Sciences, University of California - Los \\ Angeles, Los Angeles, CA, USA. \\ *correspondence e-mail address: gavindayanga@ucla.edu
}

\begin{abstract}
Global climate models produce large increases in extreme precipitation when subject to anthropogenic forcing, but detecting this human influence in observations is challenging. Large internal variability makes the signal difficult to characterize. Models produce diverse precipitation responses to anthropogenic forcing, mirroring a variety of parameterization choices for subgrid-scale processes. And observations are inhomogeneously sampled in space and time, leading to multiple global datasets, each produced with a different homogenization technique. Thus, previous attempts to detect human influence on extreme precipitation have not incorporated internal variability or model uncertainty, and have been limited to specific regions and observational datasets. Using machine learning methods, we find a physically interpretable anthropogenic signal that is detectable in all global datasets. Detection occurs even when internal variability and model uncertainty are taken into account. Machine learning efficiently generates multiple lines of evidence supporting detection of an anthropogenic signal in extreme precipitation.
\end{abstract}

Keywords: Detection and attribution, artificial neural networks, layerwise relevance propagation, Rx1day 
Introduction

Extreme precipitation can have devastating direct societal impacts such as flooding, soil

27 erosion, agricultural damages ${ }^{1}$ and indirect health risks and impacts ${ }^{2}$. Anthropogenic warming acts to

28 intensify Earth's hydrologic cycle ${ }^{3,4}$. This intensification is manifested in part through increased extreme

29 precipitation as a result of greater atmospheric moisture with warming following the Clausius-Clapeyron

30 relationship. However, circulation changes can act to enhance or reduce this increase ${ }^{4-7}$. If current

31 warming trends continue, climate models project that the Earth's atmosphere overall will move towards

32 a more intense precipitation regime ${ }^{8-11}$. Moreover, increased variation between wet and dry extremes is

33 projected, which could have devastating societal impacts ${ }^{12,13}$. These changes in extreme precipitation may have already become apparent on a regional basis ${ }^{14-17}$.

Recent studies have detected anthropogenic influence in historical changes to extreme precipitation across North America ${ }^{18}$ and Northern Hemisphere land areas $^{15}$. These attempts are part of

37 a larger category of studies known as Detection and Attribution (D\&A). Often, they initially extract the spatial or spatiotemporal patterns of climate-system response to anthropogenic forcing (so-called

39 fingerprints) from an ensemble of global climate models $(\mathrm{GCMs})^{19,20}$. Projection of observations onto

40 these fingerprints allows for signal detection as the trend of the projection ${ }^{21}$. The presence of a signal

41 that can be statistically distinguished from internal variability confirms the influence of anthropogenic

42 forcing. Thus, traditional D\&A methods rely on long term observations ${ }^{22,23}$. In the case of extreme

43 precipitation, traditional methods may be difficult to apply globally due to inordinately short records

44 and large observational uncertainty, reflected in multiple global datasets produced with very different

45 assumptions ${ }^{24-26}$. Another key difficulty with traditional methods is that the models produce a large

46 spread in the extreme precipitation response to anthropogenic forcing ${ }^{27}$. This spread occurs alongside

47 large internal variability in the models' simulations of the historical period. These two effects create 
the response have been suppressed by assuming the anthropogenic fingerprint can be derived from the

50 ensemble-mean change in extreme precipitation ${ }^{28}$. Here we aim to take these uncertainties fully into

51 account, by making no assumptions about how to derive the anthropogenic signal from GCM data.

A machine-learning-based method for the detection of anthropogenic influence (DAI) has been

53 shown to overcome the reliance on trends ${ }^{29,30}$ and is even capable of detecting the human influence

54 from weather data on a single day ${ }^{31}$. An artificial neural network (ANN) is trained to predict a proxy of

55 external forcing (e.g. the year of the data) based on the spatial maps of the target variable from an

56 ensemble of GCM simulations. Then a forced signal can be confirmed despite the presence of internal

57 climate variability and inter-model variability ${ }^{29,30}$. This ANN DAI method can identify the non-linear

58 combinations of the forced signal, internal climate variability and inter-model variability ${ }^{30}$. This method

59 also has the advantage of being able to explicitly include internal variability and model uncertainty. It

60 does not assume that any model or any model-derived quantity, such as the ensemble-mean of the

61 models, is the "true" anthropogenic signal. And it uses the raw GCM data, with GCM internal variability

62 included. In addition, ANN visualization techniques also allow for the interpretability of the models

63 formerly considered as "black boxes", making them explainable ${ }^{32,33}$, or interpretable in terms of physical

64 processes or system behavior. Use of these visualization techniques alongside the ANN DAI method

65 allows one to capture the time varying dynamic fingerprints of each input and evaluate their physical

66 credibility $^{30,34}$.

67

In this study, we apply the ANN DAI method and the ANN visualization technique known as

68 Layerwise Relevance Propagation (LRP) ${ }^{35,36}$ to global maps of annual daily maximum precipitation

69 (Rx1day) over land. Using Coupled Model Intercomparison Project, phase 5 (CMIP5) ${ }^{37}$ and phase 6

70 (CMIP6) $)^{38}$ model ensembles, we first aim to understand how the ANN is detecting the anthropogenic

71 signal and interpret it physically. Then we use the ANN to detect the anthropogenic influence on Rx1day

72 in several land-only observational and reanalysis datasets. Thus, we are agnostic about which GCM is 
correct, and which gridded data set is a true representation of the observed record. In this way we efficiently generate multiple lines of evidence as to the presence of an anthropogenic signal in the various instantiations of the observed record.

\section{Results}

ANN-identified fingerprints of anthropogenic influence

We first discuss the ability of the ANN to predict the year of occurrence for a series of simulated annual Rx1day maps. Predictions of the simulated Rx1day year (Figure 1a,b) show that the ANN struggles during roughly the first seven to eight decades of the analysis period. But prediction accuracy gradually increases, noticeably starting from the late $20^{\text {th }}$ century. Thus, compared to when this technique is applied to global-mean temperature (ref. 29) there is a lag in the emergence of the anthropogenic signal in extreme precipitation. This delay is likely due to larger internal and inter-model variability in extreme precipitation. We estimate this time of emergence (departure year) as the year when the ANN prediction continuously exceeds a selected base period (1920-1949) (see ref. 29 and 39 for more details). Some maps of simulated terrestrial Rx1day drawn from various populations of GCM training and testing data sets depart from the base period in the 1970s, but the departures mostly occur later, with lower and upper quartiles of 1991 and 2014, respectively (Figure 1c). According to the models, the anthropogenic signal has probably already emerged in Rx1day, consistent with traditional statistical methods ${ }^{40}$.

Figure $1 \mathrm{~d}$ shows the relevance pattern identified by the ANN, averaged over the period 19201949. Positive (negative) values in the relevance pattern correspond to an increase (decrease) in the predicted year. Therefore, areas of positive relevance can be interpreted as the regions with an advancing tendency on the prediction (i.e. the year) and negative values are the regions with a retreating tendency. The sum of each grid cell value is equal to the predicted year (methods, Figure 
97 S1d). These relevance patterns can be considered as the ANN-identified fingerprints of anthropogenic 98 influence (e.g. ref. 31).

The regions with positive relevance include the East Asian and African monsoon regions, and the North Pacific and Atlantic storm tracks (Figure 1d, Figure S2). The regions with negative relevance

101 include arid and semi-arid subtropical zones such as Northern African and Middle Eastern deserts, 102 Southern South Africa, Australian arid and semi-arid regions, and wet regions such as central and 103 northwestern parts of South America. Regions with negative relevance also coincide with areas 104 exhibiting a large negative dynamical component of the Rx1day trend (ref. 41, their Figure 3b). These 105 regions show a significant anthropogenic reduction of vertical velocities associated with Rx1day. This offsets the Rx1day increase stemming from the thermodynamic contribution, and produces only a weak 107 and inconsistent increase in Rx1day ${ }^{41}$. The uncertainty associated with the dynamical component has 108 been identified as a major concern for D\&A of precipitation ${ }^{42}$. As suspected, negative relevance of the 109 forced response is associated with lower signal to noise ratios than the regions with positive relevance 110 (Figure 1e,f). The signal to noise ratios are lower for both internal variability and model variability. This 111 reflects both the higher uncertainty regarding the change in extreme precipitation projected by GCMs 112 for a majority of global arid land regions, as well as larger internal variability in those regions. Here we 113 examined the time-averaged relevance patterns, but further examination of time-varying fingerprints 114 shows that the ANN is also able to identify the time-varying nature of the signal and noise 115 (Supplementary Text), which cannot be obtained directly by linear models ${ }^{34}$. The ANN-based relevance patterns are consistent with the idea that previously observed long117 term trends of terrestrial Rx1day are anthropogenic in origin (e.g. ref. 15, their Figure 1e). Many wet 118 land regions of the world have experienced a robust increase in Rx1day to date, whereas in dry regions 119 no such trend can be seen ${ }^{16,17}$. The selection of regions in these previous studies (e.g. ref. 16, 17) seems 120 to overlap with the high relevance regions in Fig.1d. 
a.

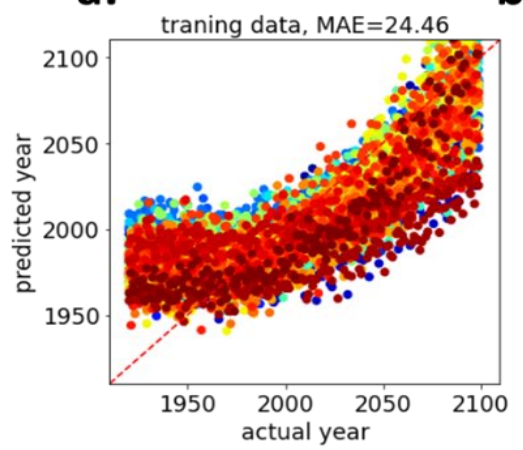

b.

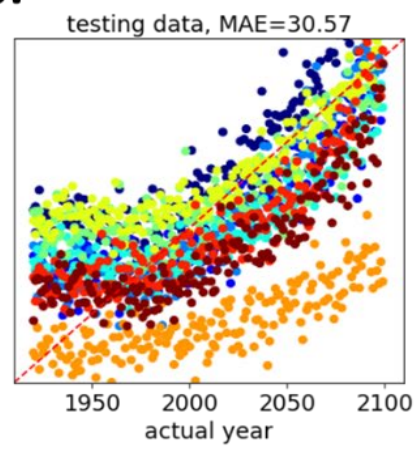

c.

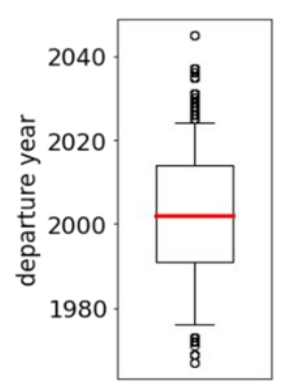

d.

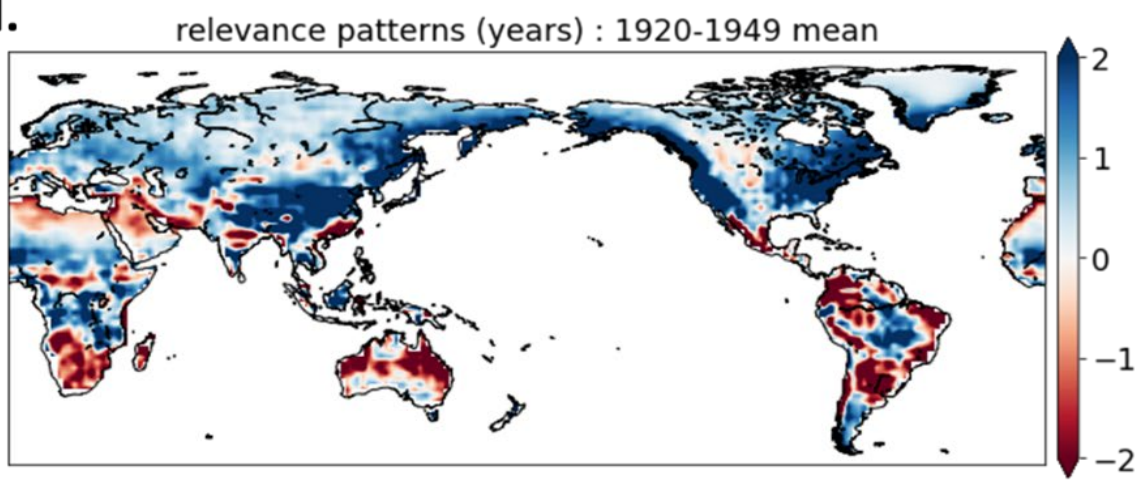

e.

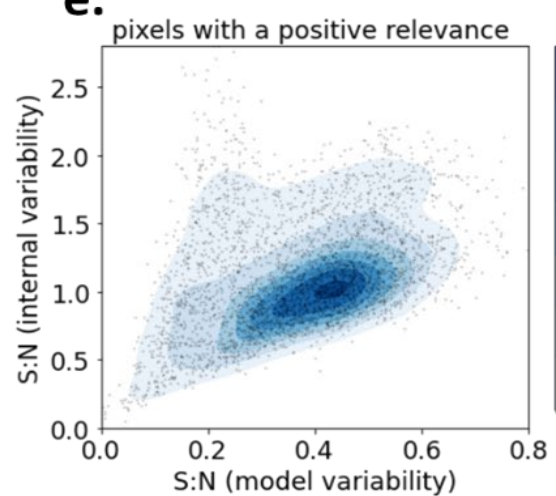

f.

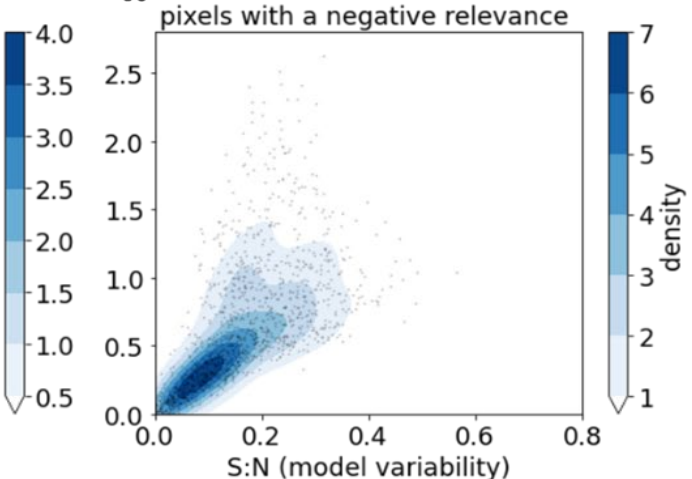

Figure 1. Fingerprint of external forcing in simulated Rx1day learned by the ANN.

$124(\mathrm{a}, \mathrm{b})$ Actual year vs predicted year for training data derived from CMIP5 and CMIP6 GCMs (a) and testing data derived from

125 CMIP5 and CMIP6 GCMs (b) for a single ANN. Each GCM is represented by a different color. (c) The year of departure from the base period, 1920-1949, obtained from 51 different ANNs with different training/testing sets. Whiskers represent the $5^{\text {th }}-95^{\text {th }}$ percentiles, while blank circles represent outliers. (d) Multimodel, ensemble-mean, layerwise-relevance-propagation-based relevance maps for Rx1day input for the period 2070-2099 from all models. (e,f) Signal to noise ratio density plots for grid cells with a positive relevance (e) and negative relevance $(f)$ in panel (d). Signal is defined as the multi-model mean change in Rx1day between the base period and 1920-1949. Noise is defined in two ways: The first stems from internal variability and is calculated 
132 model variability, and is calculated as the inter-model standard deviation of the signal from each GCM. Each dot in (e-f)

133 corresponds to one grid cell.

134

135 baseline period (1920-1949). We obtain the relevance heatmaps for each year of the baseline period for these eight models and calculate the composite difference (i.e. high value minus low value) between two sets (Fig 2a). Large positive values are seen in the African and Asian monsoon regions. The models predicting later years also have larger $20^{\text {th }}$ century mean state Rx1day values in these regions (Figure 2b). Thus, the models that predict a higher value in the baseline period have more future-like patterns of

146 Rx1day in their baseline climatologies compared to other models. When projected onto the fingerprints 147 identified by the ANN, these patterns result in a later predicted year compared to the opposite subset.

148 This exercise suggests a potential use of ANN-based DAI methods to understand how biases in historical 149 simulations project onto future changes. 

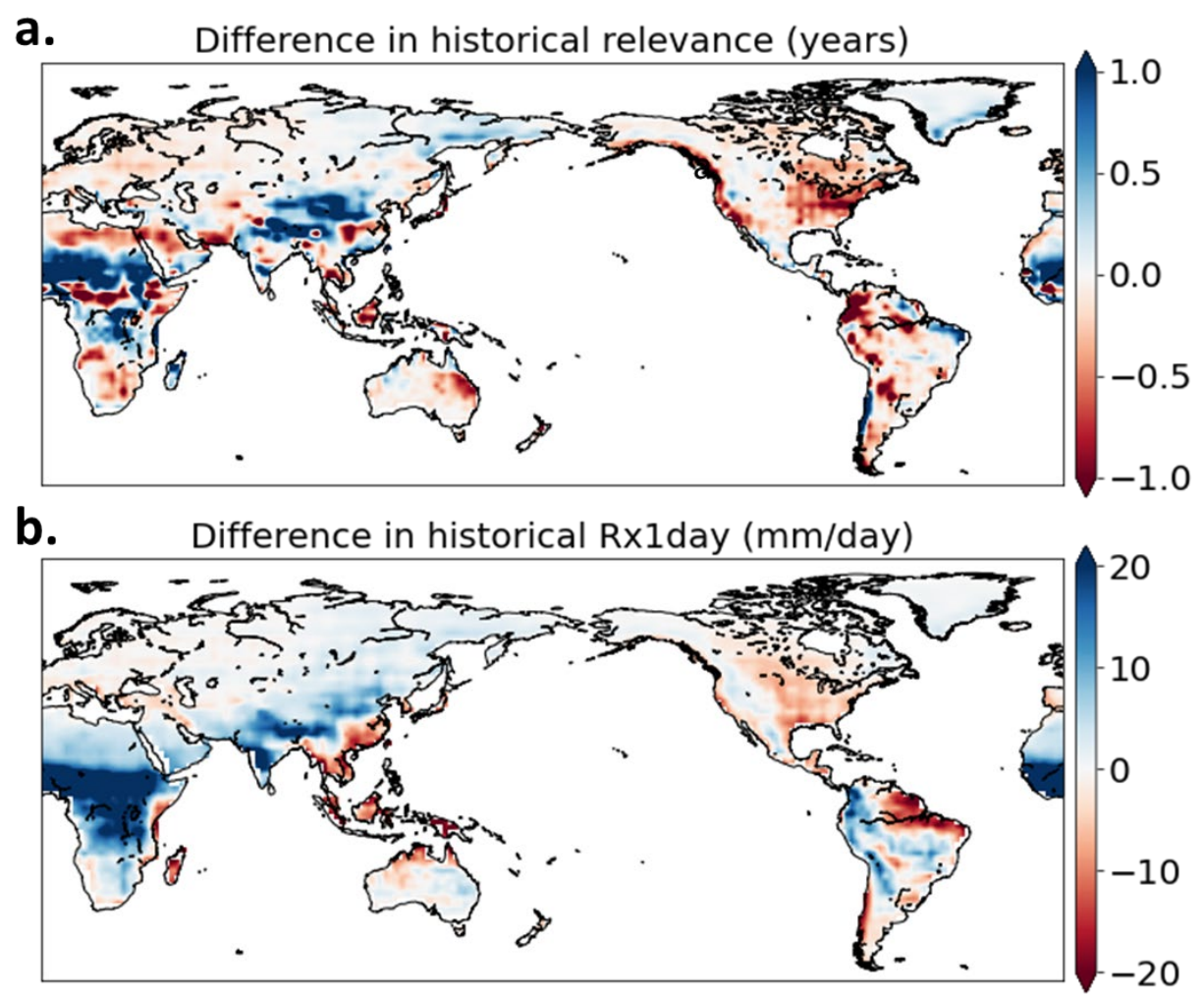

152 Figure 2. Differences between subsets of models with high and low predicted years by the ANN during the baseline period

153 (1920-1949). (a,b) The difference in their relevance maps (a) and Rx1day (b) between the four models with the highest mean

154 predicted year and the four models with lowest mean predicted year, (as shown in Figure 1a-b). The models from each subset are determined by combining the training and testing data.

\section{Detected anthropogenic signal in historical Rx1day records}

With these physical interpretations of the ANN results and relevance patterns, we use the GCM-

trained ANNs to detect whether there is a forced signal in observations. First, we calculate the globallyaveraged Rx1day trends in each dataset using a modified Mann-Kendall trend test ${ }^{43}$. Only seven out of the eleven datasets show a significant trend $(p<0.01)$ in globally-averaged Rx1day for the historical period, ranging from 0.05 to $0.09 \mathrm{~mm} /$ day/year (Table S2). Taken at face value, this suggests that the evidence for anthropogenic influence on recent changes in extreme precipitation is weak. However, 
when we apply the ANN, based on Rx1day data from GCMs, to four datasets of observational precipitation estimates and seven reanalyses, a different story emerges. time series from that dataset should have a statistically significant positive correlation with the actual year $(r)$ and a linear regression of these two variables should produce a statistically significant positive 170 slope ${ }^{21,30}$. Figure 3 shows these two metrics for observations, reanalysis and testing GCMs, from 51

171 random iterations of the ANN with different training/testing model sets. All observations and reanalysis

172 have high r values (Figure 3, Figure s3), even in datasets that do not show a significant positive trend in 173 global Rx1day record (Table S2). The $r$ values for all observational data sets are substantially larger than 174 those expected by chance (grey shaded area in Figure 3e,f). The slope is a measure of signal strength (or 175 the rate of change) in the Rx1day record. Two observational datasets (MSWEP and GPCC) are in line with 176 GCMs, along with four reanalyses (JRA55, MERRA2, NCEP2 and 20CRv3). The two REGEN datasets, ERA5 177 and CFSR show lower slopes, whereas GSWP3 has the highest slope among the datasets considered 178 here. In general, observational and renalysis products show similar correlations and slopes as the GCMs

179 for the same historical time period. (Compare the blue dots and the pink bands in Figure 3e, f.) This 180 indicates that the observational and reanalysis products show anthropogenic influence on Rx1day that is 181 comparable to what is expected from GCMs. These results demonstrate that the absence of a significant linear trend in globally averaged 183 Rx1day cannot be taken to mean there is no evidence of anthropogenic signal in Rx1day. This 184 underscores the importance of exploiting the spatial pattern of the response to external forcing to 185 extract the forced signal in observations, as opposed to the trend-based analysis ${ }^{31,34,44}$. In particular, 186 areas of negative relevance, defined previously, can act to suppress the trend in the global mean.

187 Further evidence of the importance of spatial patterns can be seen in the fact that the average ANNpredicted values vary widely and systematically across the observational datasets (Figure 3a-d, Figure 
S3). This is an indicator of systematic and large relative biases in the Rx1day climatologies of the various data sets (as pointed out above in the discussion of ANN applied to the GCMs, the average predicted value of the year depends on the magnitude of the Rx1day in the climatology (Figure 2, supplementary text)). Yet it is significant that the ANN can put the years in close to the correct order, as demonstrated by the significant correlations between actual and predicted years, even if the absolute value of the years is incorrect. This is a strong indicator that the subtle patterns and time variations of the simulated anthropogenic signal are present in the observational data sets and are shared among them, despite the fact that they are systematically biased relative to one another and likely the real world ${ }^{45,46}$.

a.

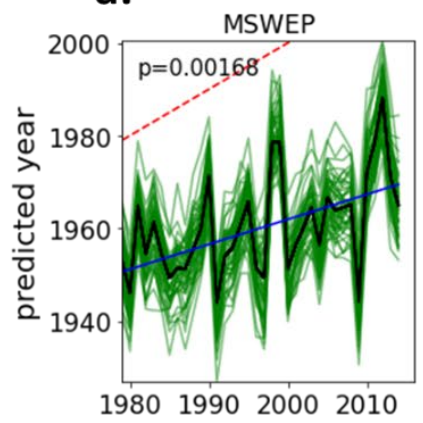

b.

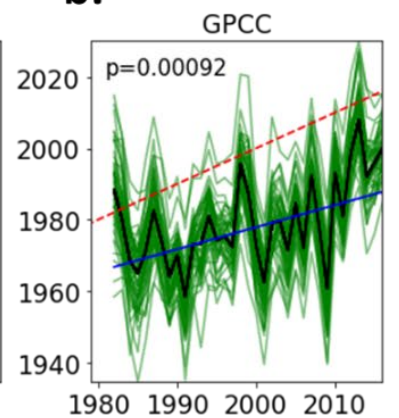

C.

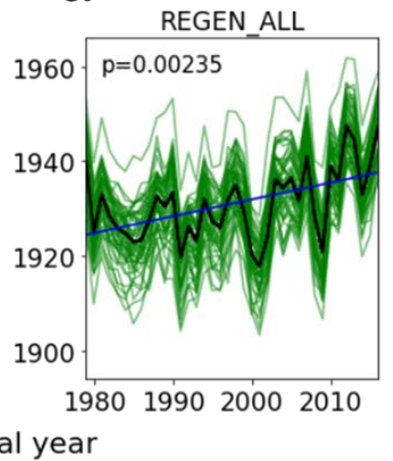

d.

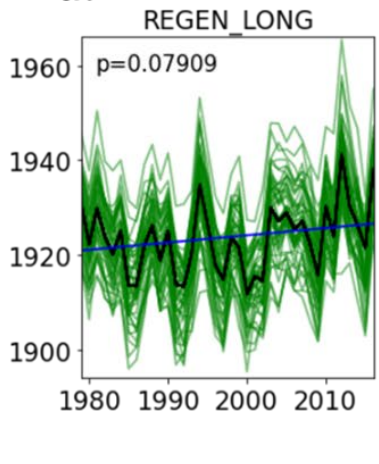

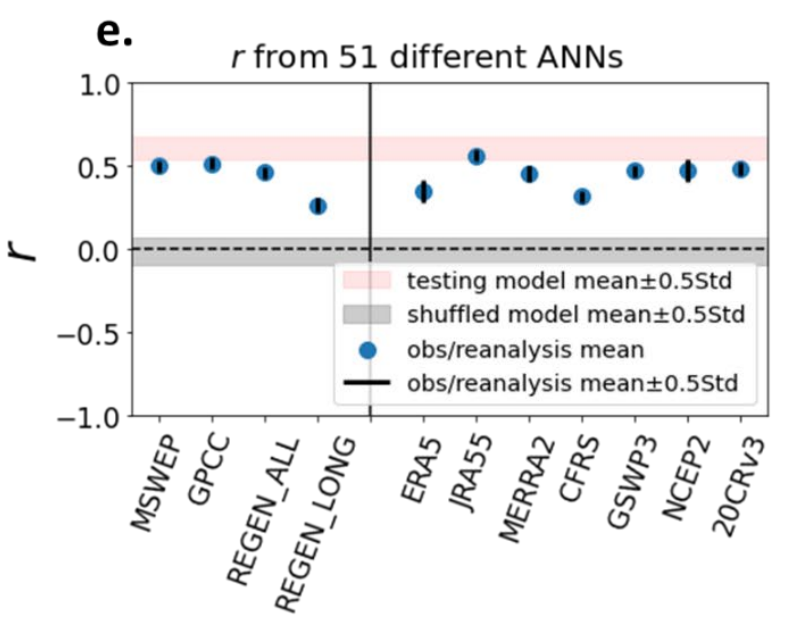

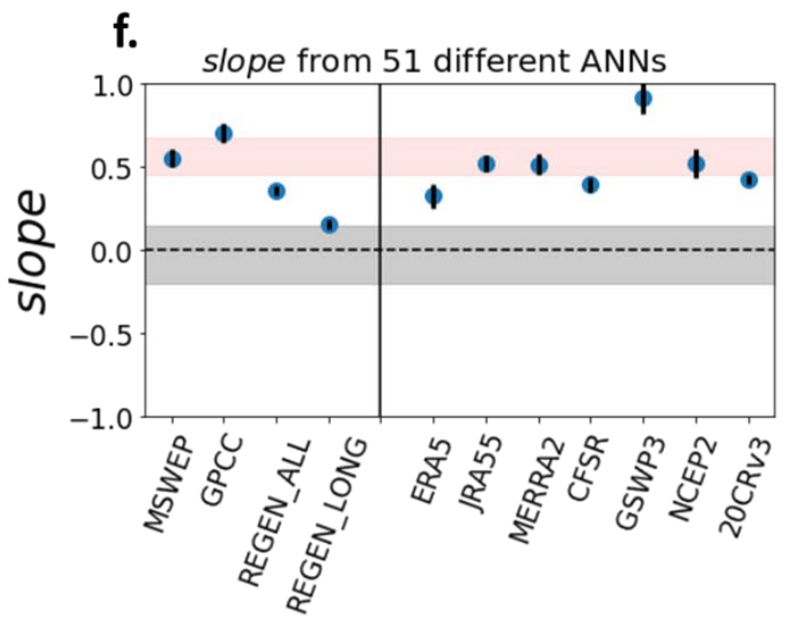

Figure 3. Metrics of the forced signal in observation-based estimates of precipitation since 1979. 
line shows the mean predicted value from all ANNs. The blue line is the best fit line of the mean predicted value. The red

dashed line is the 1:1 line. (e) Correlation ( $r$ ) between the actual years and predicted years, (f) slope of the regression line (red and grey shaded regions). Grey shading illustrates the values obtained from testing models after randomly shuffling, for each iteration of training/testing sets. Slope is obtained using the Theil-Sen method 47,48 .

\section{Conclusions}

Detecting anthropogenic signals in observations of extreme precipitation has been a challenging

211 task due to large internal variability of rare events, as well as climate model uncertainty. The limited

212 sampling in observations adds additional uncertainty, due in part to a dataset development process that

213 involves a variety of homogenization, extrapolation, and interpolation techniques to produce global

214 gridded products. Using a recently introduced ANN DAI method which utilizes the time evolution of

215 spatial maps of Rx1day in GCMs, subject to realistic radiative forcing, we find fingerprints of

216 anthropogenic signals that are physically consistent with the time evolution of the forced signal. The

217 fingerprints can be distinguished from internal variability, and emerge despite substantial model

218 uncertainty. Using this ANN DAI method, we show that the anthropogenic signal can be detected in all

219 global terrestrial Rx1day records considered in this study. This robust detection occurs despite large

220 systematic biases and large discrepancies in data sources and homogenization methods.

While previous trend-based D\&A assessments of Rx1day have demonstrated the human

222 influence in this variable in some regions, those studies assume the ensemble-mean of the GCMs is the

223 anthropogenic signal. This leads to questions as to whether further steps are needed to fully consider

224 model uncertainty ${ }^{28}$. We made a simple attempt to examine this issue by applying the ANN DAI method

225 to the same widely-used, quality-controlled Rx1day record used in the previous trend-based D\&A 
assessments. We applied the method twice, once using the same multi-model approach discussed

227 elsewhere in this study, and once assuming the ensemble-mean time series represents the true forced

228 response. Our results show that including internal variability and model uncertainty in the forced

229 response reduces the power of detection (supplementary text). Therefore, the detected signal in

230 multiple datasets in this study, with internal variability and model uncertainty being taken fully into

231 account (Figure 3 ) is a definitive affirmation of a human influence on extreme precipitation in the

232 historical record. Note that while all observations show this anthropogenic influence, the signal

233 magnitude varies considerably, on par with that seen in the GCMs. This large observational uncertainty

234 underscores a difficulty in constraining future projections of extreme precipitation with historical

235 climate model simulations and observations ${ }^{27,49}$.

236 A limitation of the ANN DAI method presented here is the inability to directly quantify the

237 detected anthropogenic influence in terms of physical units. This is especially important when

238 comparing the influence of individual external forcings (e.g. greenhouse gases, aerosols, land use and

239 land cover change, etc.). We also note that different ANN visualization techniques are available ${ }^{50}$, and

240 those should be explored to understand the sensitivity of the extracted fingerprints to the ANN

241 visualization technique. Despite these limitations, it is clear that ANN DAI methods with ANN

242 visualization techniques are very useful and efficient in identifying the human influence on variables that

243 are highly uncertain in GCMs, and poorly characterized in observations, such as extreme precipitation.

247 1. Handmer, J., et al. (2012), Changes in impacts of climate extremes: Human systems and ecosystems, 248 in Managing the Risks of Extreme Events and Disasters to Advance Climate Change Adaptation, 
edited by C. Field et al., pp. 231-290, A Special Report of Working Groups I and II of the Intergovernmental Panel on Climate Change: Managing the Risks of Extreme Events and Disasters to Advance Climate Change Adaptation, Cambridge Univ. Press, Cambridge, U. K., and New York.

2. Crimmins, A., Balbus, J., Gamble, J. L., Beard, C. B., Bell, J. E., Dodgen, D., Eisen, R. J., Fann, N., Hawkins, M. D., Herring, S. C., Jantarasami, L., Mills, D. M., Saha, S., Sarofim, M. C., Trtanj, J., \& Ziska, L. (2016). The impacts of climate change on human health in the United States: A Scientific Assessment. Washington, DC: US Global Change Research Program. https://doi.org/10.7930/JOR49NQX

3. Allen, M. R., \& Ingram, W. J. (2002). Constraints on future changes in climate and the hydrologic cycle. Nature, 419(6903), 224-232. https://doi.org/10.1038/nature01092

4. O'Gorman, P. A., and T. Schneider (2009), The physical basis for increases in precipitation extremes in simulations of 21st century climate change, Proceedings of the National Academy of Sciences of the United States of America, 106(35), 14,773-14,777, doi:10.1073/pnas.0907610106.

5. Trenberth, K. E., Dai, A., Rasmussen, R. M., \& Parsons, D. B. (2003). The changing character of precipitation. Bulletin of the American Meteorological Society, 84(9), 1205- 1218. https://doi.org/10.1175/BAMS-84-9-1205

6. Held, I. M., and B. J. Soden (2006), Robust response of the hydrological cycle to global warming, Journal of Climate, 19, 5686- 5699.

7. Norris, J., Chen, G., Neelin, J. D., Norris, J., Chen, G., \& Neelin, J. D. (2019). Thermodynamic versus dynamic controls on extreme precipitation in a warming climate from the Community Earth System Model Large Ensemble. Journal of Climate, 32, 1025-1045. https://doi.org/10.1175/JCLI-D-180302.1

8. Sun, Y., S. Solomon, A. Dai, and R. W. Portmann (2006), How often does it rain?, Journal of Climate, 19(6), 916-934, doi:10.1175/JCLI3672.1 
273 9. Fischer, E. M., U. Beyerle, and R. Knutti (2013), Robust spatially aggregated projections of climate 274 extremes, Nature Climate Change, 3(12), 1033- 1038.

275 10. Kharin, V. V., F. W. Zwiers, X. Zhang, and M. Wehner (2013), Changes in temperature and 276 precipitation extremes in the CMIP5 ensemble, Climatic Change, 119(2), 345-357, 277 doi:10.1007/s10584-013-0705-8

278 11. Sillmann, J., V. V. Kharin, F. W. Zwiers, X. Zhang, and D. Bronaugh (2013), Climate extremes indices in 279 the CMIP5 multimodel ensemble: Part 2. Future climate projections, Journal of Geophysical $280 \quad$ Research Atmospheres, 118, 2473-2493, doi:10.1002/jgrd.50188

281 12. Madakumbura, G. D., Kim, H., Utsumi, N., Shiogama, H., Fischer, E. M., Seland, Ø., Scinocca, J. F., 282 Mitchell, D. M., Hirabayashi, Y., \& Oki, T. (2019). Event-to-event intensification of the hydrologic $283 \quad$ cycle from $1.5^{\circ} \mathrm{C}$ to a $2^{\circ} \mathrm{C}$ warmer world. Scientific Reports, 9(1), 3483.

284 13. Swain, D. L., Langenbrunner, B., Neelin, J. D., \& Hall, A. (2018). Increasing precipitation volatility in 285 twenty-first-century California. Nature Climate Change, 8, 427-433. https://doi.org/10.1038/s41558-018-0140-y

287 14. Allan, R. P., and B. J. Soden (2008). Atmospheric warming and the amplification of precipitation 288 extremes, Science, 321(5895), 1481-1484, doi:10.1126/science.1160787

15. Min, S. K., X. B. Zhang, F. W. Zwiers, and G. C. Hegerl (2011), Human contribution to more-intense precipitation extremes, Nature, 470, 378-381, doi:10.1038/nature09763

291 16. Donat, M. G., A. L. Lowry, L. V. Alexander, P. A. O'Gorman, and N. Maher (2016), More extreme 292 precipitation in the world's dry and wet regions, Nature Climate Change, 6, 508- 513.

293 17. Donat, M. G., Angélil, O., \& Ukkola, A. M. (2019). Intensification of precipitation extremes in the 294 world's humid and water-limited regions. Environmental Research Letters, 14, 065003. https://doi.org/10.1088/1748-9326/ab1c8e 
precipitation in North America. Proceedings of the National Academy of Sciences of the United States of America, 117(24), 13,308-13,313, doi:10.1073/PNAS.1921628117.

19. Stott, P., N. Gillett, G. Hegerl, D. Karoly, D. Stone, X. Zhang, and F. Zwiers (2010), Detection and attribution of climate change: A regional perspective, Wiley Interdisciplinary Reviews: Climate Change, 1, 192-211, doi:10.1002/wcc.34

20. Bindoff, N. L., et al. (2013), Detection and attribution of climate change: From global to regional, in Climate Change 2013: The Physical Science Basis. Contribution of Working Group I to the Fifth Assessment Report of the Intergovernmental Panel on Climate Change, edited by T. F. Stocker et al., pp. 867-952, Cambridge Univ. Press, Cambridge, U. K., and New York.

21. Marvel, K., Cook, B. I., Bonfils, C. J., Durack, P. J., Smerdon, J. E., \& Williams, A. P. (2019). Twentiethcentury hydroclimate changes consistent with human influence. Nature, 569(7754), 59-65.

22. Hegerl, G., and F. Zwiers (2011), Use of models in detection and attribution of climate change, Wiley Interdisciplinary Reviews: Climate Change, 2(4), 570-591.

23. Easterling, D. R., Kunkel, K. E., Wehner, M. F., \& Sun, L. (2016). Detection and attribution of climate extremes in the observed record. Weather and Climate Extremes, 11, 17- 27.

24. Herold, N., Behrangi, A., \& Alexander, L. V. (2017). Large uncertainties in observed daily precipitation

26. Roca, R., Alexander, L. V., Potter, G., Bador, M., Jucá, R., Contractor, S., Bosilovich, M. G., \& Cloché,

25. Sun, Q., Miao, C., Duan, Q., Ashouri, H., Sorooshian, S., \& Hsu, K. L. (2018). A review of global precipitation data sets: Data sources, estimation, and intercomparisons. Reviews of Geophysics, 56, 79- 107. https://doi.org/10.1002/2017RG000574 S. (2019). FROGS: A daily $1^{\circ} \times 1^{\circ}$ gridded precipitation database of rain gauge, satellite and reanalysis products. Earth System Science Data, 11(3), 1017-1035. http://doi.org/10.5194/essd-11-1017-2019 
321 27. O'Gorman, P. A. (2012), Sensitivity of tropical precipitation extremes to climate change, Nature

322 Geoscience, 5(10), 697-700, doi:10.1038/NGEO1568.

323 28. Ribes, A., Zwiers, F. W., Azais, J.-M., \& Naveau, P. (2017). A new statistical approach to climate

324 change detection and attribution. Climate Dynamics, 48(1-2), 367-386.

325 29. Barnes, E. A., Hurrell, J. W., Ebert-Uphoff, I., Anderson, C., \& Anderson, D. (2019). Viewing forced

326 climate patterns through an AI Lens. Geophysical Research Letters, 46, 13,389-13,398.

327 https://doi.org/10.1029/2019GL084944

328 30. Barnes, E. A., Toms, B., Hurrell, J.W., Ebert-Uphoff, I., Anderson, C., \& Anderson, D. (2020). Indicator patterns of forced change learned by an artificial neural network. Journal of Advances in Modeling Earth Systems, 12, e2020MS002195. https://doi.org/10.1029/2020MS002195

31. Sippel, S., Meinshausen, N., Fischer, E. M., Szekely, E., \& Knutti, R. (2020). Climate change now detectable from any single day of weather at global scale. Nature Climate Change, 10, 35- 41. https://doi.org/10.1038/s41558-019-0666-7

32. Ebert-Uphoff, I., Samarasinghe, S., \& Barnes, E. (2019). Thoughtfully using artificial intelligence in Earth science. Eos, 100. https://doi.org/10.1029/2019eo135235

33. Toms, B. A., Barnes, E. A., \& Ebert-Uphoff, I. (2020). Physically interpretable neural networks for the geosciences: applications to earth system variability. Journal of Advances in Modeling Earth Systems, 12, e2019MS002002. https://doi.org/10.1029/2019MS002002

34. Wills, R.C., Sippel, S. and Barnes, E.A. (2020). Separating forced and unforced components of climate change: The utility of pattern recognition methods in Large Ensembles and observations. Variations, 18(2), pp.1-10. explanations for non-linear classifier decisions by layer-wise relevance propagation. Plos One, 10(7), e0130140. https://doi.org/10.1371/journal.pone.0130140 
36. Montavon, G., Samek, W., \& Müller, K. R. (2018). Methods for interpreting and understanding deep neural networks. Digital Signal Processing, 73, 1- 15.

37. Taylor, K. E., Stouffer, R. J., \& Meehl, G. A. (2012). An overview of CMIP5 and the experiment design. Bulletin of the American Meteorological Society, 93(4), 485- 498. https://doi.org/10.1175/BAMS-D11-00094.1

38. Eyring, V., S. Bony, G. A. Meehl, C. Senior, B. Stevens, R. J. Stouffer, and K. E. Taylor (2015), Overview of the Coupled Model Intercomparison Project Phase 6 (CMIP6) experimental design and organisation, Geoscientific Model Development Discussion, 8(12), 10,539- 10,583, doi:10.5194/gmdd-8-10539-2015

39. Mora, C., Frazier, A. G., Longman, R. J., Dacks, R. S., Walton, M. M., Tong, E. J., Sanchez, J. J., Kaiser, L. R., Stender, Y. O., Anderson, J. M., Ambrosino, C. M., Fernandez-Silva, I., Giuseffi, L. M., \& Giambelluca, T. W. (2013). The projected timing of climate departure from recent variability. Nature, 502(7470), 183-187. https://doi.org/10.1038/nature12540

40. King, A. D., Donat, M. G., Fischer, E. M., Hawkins, E., Alexander, L. V., Karoly, D. J., et al. (2015). The timing of anthropogenic emergence in simulated climate extremes. Environmental Research Letters, 10(9), 94015. https://doi.org/10.1088/1748-9326/10/9/094015

41. Pfahl, S., O'Gorman, P. A., \& Fischer, E. M. (2017). Understanding the regional pattern of projected future changes in extreme precipitation. Nature Climate Change, 7(6), 423-427. https://doi.org/10.1038/nclimate3287

42. Shepherd, T. G. (2014), Atmospheric circulation as a source of uncertainty in climate change projections, Nature Geoscience, 7, 703-708, doi:10.1038/ngeo2253

43. Hamed, K. H., and A. R. Rao (1998), A modified Mann-Kendall trend test for autocorrelated data, Journal of Hydrology, 204, 182- 196.

44. Wills, R. C. J., Battisti, D. S., Armour, K. C., Schneider, T., \& Deser, C. (2020). Pattern Recognition 
Methods to Separate Forced Responses from Internal Variability in Climate Model Ensembles and Observations, Journal of Climate, 33(20), 8693-8719. https://doi.org/10.1175/JCLI-D-19-0855.1

371

45. Bador, M., Alexander, L. V, Contractor, S., \& Roca, R. (2020). Diverse estimates of annual maxima daily precipitation in 22 state-of-the-art quasi-global land observation datasets. Environmental Research Letters, 15(3), 35005. http://doi.org/10.1088/1748-9326/ab6a22

46. Alexander, L. V., Bador, M., Roca, R., Contractor, S., Donat, M., \& Nguyen, P. (2020). Intercomparison of annual precipitation indices and extremes over global land areas from in situ, space-based and reanalysis products. Environmental Research Letters, 15(5), 055002. https://doi.org/10.1088/1748-9326/ab79e2

47. Theil, H. (1950), A rank-invariant method of linear and polynomial regression analysis, Indagationes

48. Sen, P. K. (1968), Estimates of the regression coefficient based on Kendall's tau. Journal of the Mathematicae, 12, 85-91.

49. Borodina, A., Fischer, E. M., \& Knutti, R. (2017). Models are likely to underestimate increase in heavy rainfall in the extratropical regions with high rainfall intensity. Geophysical Research Letters, $\mathbf{4 4}$, 7401- 7409. https://doi.org/10.1002/2017GL074530

50. Samek, W., Montavon, G., Vedaldi, A., Hansen, L. K., \& Müller, K. R. (Eds.). (2019). Explainable Al: Interpreting, explaining and visualizing deep learning. Springer Nature.

\section{Data and Methods}

Data

We use daily precipitation rate output from a collection of climate models participating in 
to 2099 for each model. Future projections from CMIP5 follow the Representative Concentration

394 Pathway 8.5 (RCP 8.5) $)^{51}$, while CMIP6 projections follow the Shared Socioeconomic Pathway 5-8.5 (SSP

$3955-8.5)^{52}$. To increase our sample size, we combine both CMIP5 and CMIP6 model subsets into one

396 ensemble, which is justifiable considering the very similar time evolution of the total anthropogenic

397 forcing in RCP 8.5 and SSP 5-8.5 scenarios (ref. 52, their Figure 3c). We regrid all daily precipitation data 398 to a $2^{\circ} \times 2^{\circ}$ spatial grid and compute the Rx1day value for each year at each land grid point.

We use four datasets of observational estimates of daily precipitation rate with global

400 coverage: Multi-Source Weighted-Ensemble Precipitation, version 2 (MSWEP) ${ }^{53}$, Global Precipitation

401 Climatology Centre (GPCC) version 2018 ${ }^{54}$, and Rainfall Estimates on a Gridded Network (REGEN) ${ }^{55}$, 402 including both REGEN_ALL and REGEN_LONG . MSWEP is a hybrid reconstruction using in situ, satellite 403 and reanalysis data, whereas GPCC and the REGEN datasets are developed from ground-based 404 measurements. REGEN_ALL is developed by interpolating all considered station data whereas 405 REGEN_LONG is developed using only the stations with a data record of 40 years or longer. We further 406 use seven widely used reanalysis products for comparison : ECMWF ERA $5^{56}$, Japanese 55-year Reanalysis $407 \quad(\text { JRA55) })^{57}$, Modern-Era Retrospective analysis for Research and Applications, Version 2 (MERRA2) ${ }^{58}$, 408 NCEP Climate Forecast System Reanalysis (CFSR) ${ }^{59}$, Global Soil Wetness Project Phase $3\left(\right.$ GSWP3) ${ }^{60}$, 409 NCEP-DOE Reanalysis 2 (NCEP2) ${ }^{61}$ and NOAA-CIRES-DOE Twentieth Century Reanalysis version 3 $410(20 \mathrm{CRv} 3)^{62}$. These observational and reanalysis datasets are selected considering the availability of full 411 global land coverage and data for at least three decades (Table S2). All observation and reanalysis data 412 were regridded to the same $2^{\circ} \times 2^{\circ}$ spatial grid as the models, and then Rx1day was calculated at each 413 grid point for each year. 
Here we apply the method in ref. 29 (see their Figure 1a) to predict the year with which given

417 annual Rx1day maps from GCMs are associated, a regression task. This requires the ANN to learn the 418 signature of the forced response in simulated Rx1day. By feeding the ANN multicentury data from 419 forced simulations, it learns to distinguish the forced signal from internal climate variability. The use of 420 multiple GCMs helps the ANN learn the common elements of the forced response most relevant to the 421 prediction task, a process that fully considers model uncertainty as well as internal climate variability.

422 Input to the ANN from each model is a vectorized spatial map of Rx1day $\left(2^{\circ} \times 2^{\circ}\right.$ spatial grid $=16200$ grid 423 values) for each year from 1920 to 2099. Our primary goal is to detect the anthropogenic signal in 424 extreme precipitation over land. Thus, we mask out data over the ocean at this stage, resulting in 6082 425 land grid values. The ANN architecture consists of two hidden layers with ten nodes each. The Rectified 426 Linear Unit activation function is used for all hidden units. Approximately $80 \%$ of the models (35) are used for training the ANN, while the rest (9) are used 428 for testing. K-fold validation is applied to split the initial training dataset into training and validation 429 datasets. Here we set $\mathrm{K}=2$. The mean squared error between the actual and predicted year of Rx1day is 430 used as the loss function to be minimized during the training. For the optimizer which updates the ANN 431 based on the gradient of the loss, we select rmsprop. Climate variables inherently contain spatial 432 autocorrelation. To account for this dependence among adjacent input data points, we use L2 433 regularization between inputs and the first hidden layer, which adds the sum of squared weights as a 434 penalty term to the loss function. By iterating over L2 values of leading order of magnitudes and 435 inspecting the tradeoff between low prediction error and generalizability (Figure S1), we found L2=0.001 436 to be a suitable value for our analysis.

We could have made the ANN more complex to achieve higher accuracy. But we elected not to 438 do so, partly because Increasing the number of hidden units or changing the other hyperparameters 439 (except for L2 regularization) did not result in a substantial increase in accuracy. More importantly, we 
aimed to keep the ANN simple, with a reasonable degree of accuracy. This is because the main goal is

441 not to obtain a perfect prediction, but rather to reveal the forced patterns the ANN learns (e.g. ref. 29,

$44230)$. As we show in Section 3, imperfections in the prediction also can be physically interpreted within

443 the D\&A research framework (Figure 2).

444

445

Neural network interpretation using Layerwise Relevance Propagation (LRP)

Assume that for a given input map, $\boldsymbol{x}$, we get an output $f(x)$, in our case, the predicted year.

447

LRP conservatively back-propagates this value through hidden layers until it reaches the input map. This

448 process generates a relevance heatmap, indicating the areas of importance influencing the value $f(x)$.

449 The conservation property is shown in eq.1, for relevance propagation between two hidden layers $j$ and

$450 k$, where $k$ is the higher layer (i.e. closer to the output). The summation operation for each layer (e.g.

$\left.451 \sum_{k} P_{k}\right)$ is the summation of the relevance $(P)$ of all hidden units in that layer. The activation, $a_{k}($ eq. 2$)$ is

452 the information coming from all units in layer $j$, to a target unit in layer $k$. In eq. $2, a_{j}$ values are the

453 individual activations of each unit in the layer $j, w_{j k}$ values are the weights associated with the

454 relationship between each unit in layer $j$ and the target unit $k$, and $b_{k}$ is the bias of that target unit.

455

456

$$
\sum_{i=1}^{d} P_{i}=\cdots=\sum_{j} P_{j}=\sum_{k} P_{k}=\cdots=f(x)
$$

457

458

$$
a_{k}=\operatorname{ReLu}\left(\sum_{j} a_{j} w_{j k}+b_{k}\right)
$$

459

460

$$
P_{j}=\sum_{k}\left(\alpha \frac{a_{j} w_{j k}^{+}}{\sum_{j} a_{j} w_{j k}^{+}}-\beta \frac{a_{j} w_{j k}^{-}}{\sum_{j} a_{j} w_{j k}^{-}}\right)
$$


The relevance propagation rule from layers $j$ to $k$ is given in eq. 3. This general form is also

463 known as the $\alpha \beta$-rule $\mathrm{e}^{35,36}$. The components ()$^{+}$and ()$^{-}$indicate only positive and negative weights are

464 being considered, respectively. The $\alpha$ and $\beta$ coefficients represent the relative amount of positive and

465 negative relevance to be propagated, respectively. As shown in eq. 3, positive relevance (i.e. excitatory

466 influence) and negative relevance (i.e. inhibitory influence) are associated with positive and negative

467 weights, respectively. The $\alpha$ and $\beta$ coefficients are to be chosen with the constraints $\alpha-\beta=1$ and $\beta \geq$

4680 . The combination $\alpha=2$ and $\beta=1\left(\mathrm{LRP}_{\alpha 2 \beta 1}\right)$ have been experimentally inferred as suitable values,

469 and have been adopted in previous research ${ }^{35,36,63-65}$. Here we adopt the $\operatorname{LRP}_{\alpha 2 \beta 1}$ rule.

470 The $\alpha \beta$-rule rule with $\alpha=1$ and $\beta=0$ (LRP $\alpha 1 \beta 0$, also known as the Deep Taylor Decomposition)

471 is a special case where for each input, the sum of LRP relevance heatmaps is equivalent to $f(x)$. This is a

472 valuable property for the interpretation of results. As $\beta=0, \mathrm{LRP}_{\alpha 1 \beta 0}$ only considers the information

473 which positively contributes to the final decision. For regression tasks such as the problem at hand here,

474 inputs which contribute to a decrease in $f(x)$ (i.e. an earlier predicted year; negative relevance) are

475 equally as important as inputs which contribute to an increase (i.e. a later predicted year; positive

476 relevance) to understand what the ANN has learned. Moreover, when $\alpha>1$, the $\alpha \beta$-rule does not

477 conserve the relevance from the output value back to the input layer. For these reasons, ref. 33 pointed

478 out that caution should be exercised when applying the $\alpha \beta$-rule with 1) $\alpha=1$ for regression and 2) $\alpha>$

4791 in general. We find that for our simple $A N N$, applying $L R P_{\alpha 2 \beta 1}$ results in a 1:1 relationship between the

480 resultant relevance heatmaps and $f(x)$ for each input (Figure S1d). This allows the visualization of input

481 that contributes to a decrease in $f(x)$ while maintaining a direct relationship between the ANN

482 predicted value and LRP heatmaps. Therefore, we proceed with rescaled relevance heatmaps derived

483 from LRP $\alpha_{\alpha 2 \beta 1}$ for interpreting our ANN. More details on LRP can be found in previous work (ref.

$48433,35,36,66)$. 
51. Meinshausen, M., et al. (2011), The RCP greenhouse gas concentrations and their extensions from 1765 to 2300, Climatic Change, 109(1-2), 213-241, doi:10.1007/s10584-011-0156-z

52. O'Neill, B. C., Tebaldi, C., Van Vuuren, D. P., Eyring, V., Friedlingstein, P., Hurtt, G., Knutti, R., Kriegler, E., Lamarque, J. F., Lowe, J., Meehl, G. A., Moss, R., Riahi, K., \& Sanderson, B. M. (2016). The

53. Beck, H. E., Wood, E. F., Pan, M., Fisher, C. K., Miralles, D. G., van Dijk, A. I., \& Adler, R. F. (2018). MSWEP V2 global 3-hourly $0.1^{\circ}$ precipitation: Methodology and quantitative assessment. Bulletin of the American Meteorological Society, 100, 473- 500. https://doi.org/10.1175/BAMS-D-17-0138.1

54. Ziese, M, Rauthe-Schöch, A, Becker, A, Finger, P, Meyer-Christoffer, A, \& Schneider, U. (2018). GPCC

55. Contractor, S., Donat, M. G., Alexander, L. V., Ziese, M., Meyer-Christoffer, A., Schneider, U., full data daily version. 2018 at $1.0^{\circ}$ : Daily land-surface precipitation from rain-gauges built on GTSbased and historic data: Global Precipitation Climatology Centre (GPCC, http://gpcc.dwd.de/) at Deutscher Wetterdienst. Rustemeier, E., Becker, A., Durre, I., \& Vose, R. S. (2020). Rainfall Estimates on a Gridded Network (REGEN)-A global land-based gridded dataset of daily precipitation from 1950-2013. Hydrology and Earth System Sciences, 24(2), 919-943. https://doi.org/10.5194/hess-24-919-2020

56. Hersbach, H., Bell, B., Berrisford, P., Hirahara, S., Horanyi, A., Munoz-Sabater, J., Nicolas, J., Peubey, C., Radu, R., Schepers, D., Simmons, A., Soci, C., Abdalla, S., Abellan, X., Balsamo, G., Bechtold, P., Biavati, G., Bidlot, J., Bonavita, M., Chiara, G., Dahlgren, P., Dee, D., Diamantakis, M., Dragani, R., Flemming, J., Forbes, R., Fuentes, M., Geer, A., Haimberger, L., Healy, S., Hogan, R. J., Holm, E., Janiskova, M., Keeley, S., Laloyaux, P., Lopez, P., Lupu, C., Radnoti, G., Rosnay, P., Rozum, I., Vamborg, F., Villaume, S., \& Thepaut, J.-N. (2020). The ERA5 global reanalysis. Quarterly Journal of 
the Royal Meteorological Society, 2020, 1- 51. https://doi.org/10.1002/qj.3803

511 57. Kobayashi, S., et al. (2015), The JRA-55 reanalysis: General specifications and basic characteristics, Journal of the Meteorological Society of Japan, 93(1), 5- 48, doi:10.2151/jmsj.2015-001

58. Gelaro, R., McCarty, W., Suárez, M. J., Todling, R., Molod, A., Takacs, L., ... Zhao, B. (2017). The Modern-Era Retrospective Analysis for Research and Applications, Version 2 (MERRA-2). Journal of

59. Saha, S., S. Moorthi, H.-L. Pan, and 49 others (2010), The NCEP climate forecast system reanalysis, Bulletin of the American Meteorological Society, 91(8), 1015- 1057, doi:10.1175/2010BAMS3001.1

60. Kim, H. (2017), Global Soil Wetness Project Phase 3 Atmospheric Boundary Conditions (Experiment 1), Data Integration and Analysis System (DIAS). https://doi.org/10.20783/DIAS.501

61. Kanamitsu, M., W. Ebisuzaki, J. Woollen, S.-K. Yang, J. Hnilo, M. Fiorino, and G. L. Potter (2002), NCEP-DOE AMIP-II reanalysis (R-2), Bulletin of the American Meteorological Society, 83, 1631- 1643.

62. Slivinski, L. C., Compo, G. P., Whitaker, J. S., Sardeshmukh, P. D., Giese, B. S., McColl, C., Allan, R., Yin, X., Vose, R., \& Titchner, H. (2019). Towards a more reliable historical reanalysis: Improvements for version 3 of the Twentieth Century Reanalysis system. Quarterly Journal of the Royal Meteorological

64. Dobrescu, A., Valerio Giuffrida, M., \& Tsaftaris, S. A. (2019). Understanding deep neural networks for regression in leaf counting, Proceedings of the IEEE Conference on Computer Vision and Pattern

63. Böhle, M., Eitel, F., Weygandt, M., \& Ritter, K. (2019). Layer-wise relevance propagation for Society, 145(724), 2876- 2908. Recognition Workshops (pp. 2600- 2608). Long Beach, CA, USA: IEEE.

65. Grigorescu, I., Cordero-Grande, L., Edwards, A. D., Hajnal, J. V., Modat, M., \& Deprez, M. (2019). Investigating Image Registration Impact on Preterm Birth Classification: An Interpretable Deep 

Analysis, pp. 104-112, Springer, Cham. propagation: an overview. In Explainable Al: interpreting, explaining and visualizing deep learning (pp. 193-209). Springer, Cham. doi: 10.1007/978-3-030-28954-6_10.

\section{Acknowledgements}

542 We also acknowledge the World Climate Research Programme's Working Group on Coupled Modelling,

543 which is responsible for CMIP, and we thank the climate modelling groups for producing and making

544 available their model output. We also thank the Earth System Grid Federation (ESGF) for archiving the

545 data and providing access, and various funding agencies who support CMIP and ESGF. Funding: We

546 acknowledge support from the Regional and Global Model Analysis Program for the Office of Science of

547 the U.S. Department of Energy through the Program for Climate Model Diagnosis and Intercomparison.

548 Competing financial and non-financial interests: The authors declare no competing interests. Data and

549 materials availability: Data and materials that support the findings of this study are available upon

550 reasonable request from the authors. 